\title{
Temperature dependence of the exciton gap in monocrystalline $\mathrm{CuGaS}_{2}$
}

\author{
S. Levcenco ${ }^{a, *}$, S. Doka ${ }^{b}$, V. Tezlevan ${ }^{a}$, D. Fuertes Marron ${ }^{c}$, L. Kulyuk ${ }^{a}$, T. Schedel-Niedrig ${ }^{b}$, \\ M. Ch.Lux-Steiner ${ }^{b}$, E. Arushanov ${ }^{a, b}$ \\ 'Institute of Applied Physics, Academy of Sciences of Moldova, Chisinau, MD 2028, Republic of Moldova \\ bahn-Meitner institut GmbH, Glienicker Straße 100, D-14i09 Berin, Germany \\ ' instituto de Energía Solar - ETST, Universidad Politéctica de Madid, Cudad Universitaria s.n., 28040, Spain
}

\section{A R T I C L. E I N F O}

Keywords:

Chalcopyrite

Photoluminescence Spectroscopy

\begin{abstract}
A B S T R A C T
Single crystals of $\mathrm{CuGaS}_{2}$ have been grown by chemical vapour transport. Their near-band gap photoluminescence properties were investigated in the temperature range of $10-300 \mathrm{~K}$. The variation of the exciton gap energy with temperature was studied by means of a three-parameter thermodynamic model, the Einstein model and the Pässler model. Values of the band gap at $T=0 \mathrm{~K}$, of a dimensionless constant related to the electron-phonon coupling, and of an effective and a cut-off phonon energy have been estimated. It has also been found that the major contribution of phonons to the shift of $E_{g}$ as a function of $\mathrm{T}$ in $\mathrm{CuGaS}_{2}$ is mainly from optical phonons.
\end{abstract}

\section{Introduction}

CulnSe $\mathrm{Se}_{2}$ and related chalcopyrite-type semiconductors are leading candidates for absorbers in high-efficiency heterojunction solar cells. Devices based on this material have demonstrated efficiences up to $19.9 \%$ [1]. Although not optimally suited as an absorber for single-gap solar cells due its high band gap $\mathrm{CuGaS}_{2}$ has been proposed for optoelectronic applications [2]. So far, the characteristics of $\mathrm{CuGaS}_{2}$ single crystals have not been yet well studied. Some optical measurements (absorption, reflectivity spectra, pholuminescence and photoreflectance, Raman scattering and spectroscopic ellipsometry) were carried out on $\mathrm{CuGaS}_{2}$ thin films and bulk samples [3-12].

However, only one work devoted to the analysis of the temperature-dependence of the band gap in $\mathrm{CuGaS}_{2}$ over a wide range of temperatures has been reported [6]. For nearly stoichiometric $\mathrm{CuGaS}_{2}$ epitaxial layers and bulk $\mathrm{CuGaS}_{2}$ crystal, the temperature-dependence of the near-band-edge PL has been fitted according to the Einstein model, from which the Einstein characteristic temperature and the free-exciton energy at $0 \mathrm{~K}$ were determined.

In this study we report on temperature-dependent, steadystate photoluminescence measurements on $\mathrm{CuGaS}_{2}$ single crystals grown by chemical vapour transport. Single crystals are used as a reference system to obtain reliable data on the physical properties of the material and its parameters, excluding the effects of substrate stress and extrinsic doping due to diffusion (likely in

\footnotetext{
* Corresponding autor.

E-mail address: levcencoenandex.ru (S. Levcenco).
}

thin films grown on foreign substrates). The value of the exciton gap is determined from luminescence measurements and its variation with temperature is studied using three different models, namely a three-parameter thermodynamic model of O'Donnell and Chen [13], the Einstein model [14] and the Pässler mode] [15].

\section{Experimental methods}

Single crystals were grown by $\mathrm{CVT}$ in a closed system using iodine as a transport agent. As-grown crystals have a plate-like form. The monocrystallinity of the crystals was proved by the Laue X-ray diffraction method.

Photoluminescence spectra were obtained between $10 \mathrm{~K}$ and room temperature using the $457.9 \mathrm{~nm}$ line of $\mathrm{a} \mathrm{cW} \mathrm{Ar}^{+}$as the excitation source. Luminescence was spectrally analysed by a grating monochromator and a Hamamatsu photomultiplier.

\section{Results and discussions}

In Fig. 1, temperature dependence of PL spectra is shown for nearly stoichiometric $\mathrm{CuGaS}_{2}$ single crystal. Exciton peaks at $2.500,2.491$, and $2.483 \mathrm{eV}$ are observed at $10 \mathrm{~K}$. The former two peaks could be related to free excitons or polaritons, while the peak at $2.483 \mathrm{eV}$ is the exciton bound to neutral acceptor [6]. The above named excitonic peaks do not show appreciable temperature dependence below $70 \mathrm{~K}$. However intensity of the bound exciton decreases as temperature increases from 10 to $70 \mathrm{~K}$. The high temperature luminescence spectra $(>70 \mathrm{~K})$ 


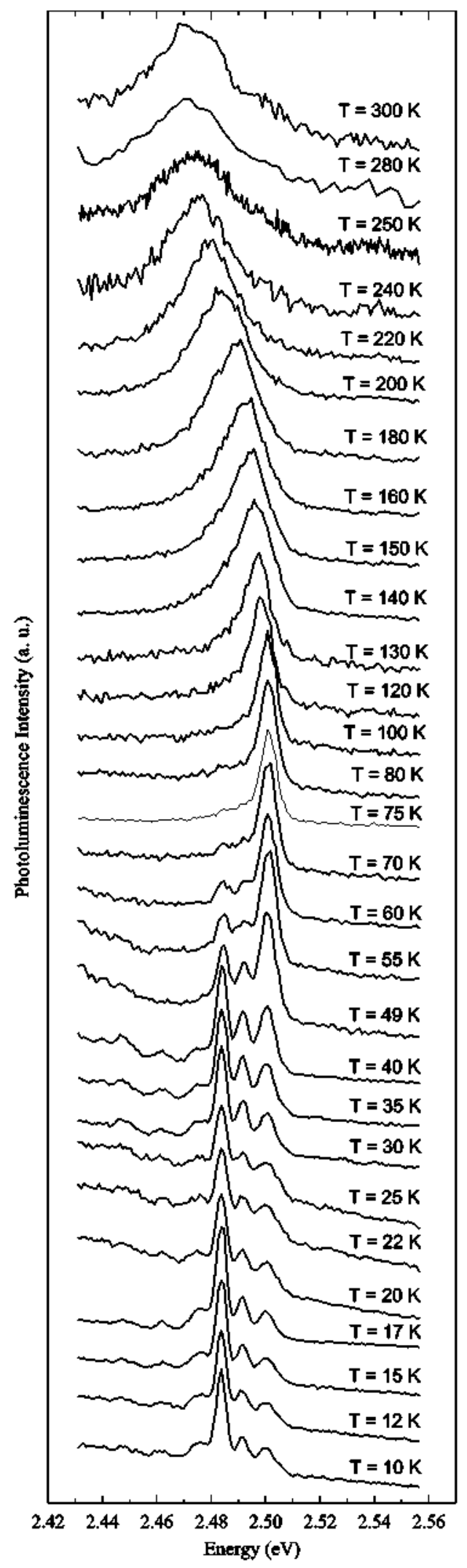

Fig. 1. Temperature dependence of the near-band-edge PL spectra for bulk CuGaS single crystal.

presented by free exctiton, which is broadened and thermally dissolved up to room temperature. The observed near-band gap $\mathrm{PL}$ is in a good agreement with the PL properties of epitaxial $\mathrm{CuGaS}_{2}$ layers on $\mathrm{Si}\left(\begin{array}{lll}1 & 1 & 1\end{array}\right)[8,12]$, GaAs and $\mathrm{GaP}[6]$ but in contrast to the bulk $\mathrm{CuGaS}_{2}$ where bound exciton peak is dominated at high temperature PL spectra [6]. The latter behavior of the reported $\mathrm{PL}$ on bulk $\mathrm{CuGaS}_{2}$ is probably due to some imperfections of the crystal and/or presence of defects in the studied samples.

The value of the exciton gap is determined from luminescence measurements and its variation with temperature is studied using three different models, the three-parameter thermodynamic model of O'Donnell and Chen [13], the Einstein model [14| and the Pässler model [15]. The physical bases of the three models are briefly summarized [16|. For many semiconductors the contribution of the electron-phonon coupling to the temperature variation of the band gap energy is dominant. A three-parameter thermodynamic model [13] can be described as:

$\left.E_{\mathrm{g}}(T)=E_{\mathrm{g}}(0)-S\left(E_{\mathrm{ph}}\right)\left[\operatorname{coth}\left\langle E_{\mathrm{ph}}\right\rangle / 2 k_{\mathrm{B}} T\right)-1\right]$,

where $E_{\mathrm{g}}(0)$ is the band gap at $T=0 K,\left\langle E_{\mathrm{ph}}\right\rangle$ is an effective phonon energy, and $S$ is a dimensionless constant related to the electron-phonon coupling. Eq. (1) provides a good description of $E_{\mathrm{g}}(T)$ for various semiconducting materials such as Si, GaAs, GaP, diamond [13], $\mathrm{CuGaSe}_{2}$ [16], $\beta-\mathrm{FeSi}_{2}$ [17], $\mathrm{CuGa}_{3} \mathrm{Se}_{5}$ [18] and $\mathrm{CuIn}_{5} \mathrm{Se}_{8}$ [19].

Using the Einstein model, the $E_{g}(T)$ dependence can be expressed by the equation [14]

$E_{\mathrm{g}}(T)=E_{\mathrm{g}}(0)-K\left(e^{\bar{z} / T}-1\right)^{-1}$,

where $K$ is a temperature-independent constant and $\Xi$ is the Einstein temperature. Based on the equality $\operatorname{coth}(x / 2)-1=$ $2 /(\exp (x)-1)$, Pässler [15] showed the identity of the empirical Eqs. (1) and (2) simply defining $x=\left\langle E_{\mathrm{ph}}\right\rangle / K_{B} T$. Then Eq. (1) can be rewritten in the following manner:

$E_{g}(T)=E_{g}(0)-\left(2 S\left\langle E_{\mathrm{ph}}\right\rangle\right) /\left(\left(\exp \left\langle E_{\mathrm{ph}}\right\rangle / k_{\mathrm{B}} T\right)-1\right)$.

Eq. (3) represents the Einstein model (2) with the Einstein temperature $\bar{E}=\left\langle E_{p h}\right\rangle / K_{B}$ and the constant $K=2 S\left\langle E_{p h}\right\rangle$.

Pässler [15| proposed an analytical description of $E_{\mathrm{g}}$ versus $T$ within the regime of dominant electron-phonon interaction. According to this theory, the variation can be expressed by

$E_{g}(T)=E_{g}(0)-\int f(\varepsilon)(1 / 2+n(\varepsilon, T)) d \varepsilon$,

where $\varepsilon$ is the phonon energy, $n(\varepsilon, T)=\left[\exp \left(\varepsilon / k_{\mathrm{B}} T\right)-\left.1\right|^{-1}\right.$ represents the average phonon occupation number, and $f(\varepsilon)$ is the relevant electron-phonon spectral function, which is given by a power-law dependence of the form

$f(\varepsilon) \propto \varepsilon^{v}$

within the energy range of $0 \mathrm{eV}$ up to a cut-off value of $\varepsilon_{\mathrm{c}}=[(v+1)]$ $\nu \mid k_{\mathrm{B}} \Theta$. For exponents within a range of $1.2<v<1.6$, which correspond to a moderately concave spectral function, Eq. (4) can be simplified to [15]

$E_{g}(T)=E_{g}(0)-\alpha(\Theta / 2)\left\{\left[1+(2 T / \Theta)^{p}\right]^{1 / D}-1\right\}$,

where $\delta$ is equal to the high-temperature limit of the associated entropy, $\Theta$ is the effective phonon temperature (expected to be smaller than the Debye temperature, $2 \theta_{\mathrm{D}} / 3$ [15]), and the parameter $p=v+1$. The exponent $\eta$ governs the shape of the spectral function.

A spectral function of the power-law type (4a) is not the only way of modelling of concave behavior [15]. A rather informative alternative ansatz is given by a function of type [15]

$f(\varepsilon)=C \varepsilon /\left(\varepsilon_{c}-\varepsilon\right)^{\delta}$.

Up to a certain cut-off energy $\varepsilon_{c}=(2-\delta) k_{B} \Theta$ (and $=0$ elsewhere). The actual shape of this model function is controlled by a parameter $0<\delta<1$, which (similar to $p$ in (5a)) may be estimated from experimental results by fitting the measured $E_{g}(T)$ curve. The $E_{g}(T)$ dependence is given by an analytical representation of the 
form

$E_{g}(T)=E_{g}(0)-\alpha(\Theta / 2)\left\{\left[1+2 r(2 T / \Theta)^{2}+(2 T / \Theta)^{4}\right]^{1 / 4}-1\right\}$

where the parameter $r$ is connected with the parameter $\delta$ by

$r=(1-\delta) \pi^{2} /(2-\delta) 6$ i.e. conversely $\delta=\left(\pi^{2}-12 r\right) /\left(\pi^{2}-6 r\right)$.

Eq. (7) represents a reasonable approximation at least for $\delta<0.9$ i.e $r>0.15$, which is sufficient for most applications [15]. It is also shown from both theoretical considerations and practical applications that both analytical Eg (T) model expressions (5) and (7) are nearly equivalent [15]. Nevertheless, particularly for the sake of numeral consistency of the tests, it may be useful to apply in practice both competitive representations simultaneously, particularly in cases of wide-gap materials [15].

From Eqs. (1) or (2), it can be written [16]

$\mathrm{d} E_{g} / \mathrm{d} T=-S\left\langle E_{\text {ph }}^{2}\right\rangle / 2 K_{B} T^{2} \operatorname{Sinh}^{2}\left(\left\langle E_{\mathrm{ph}}\right\rangle / 2 K_{B} T\right)$.

At high temperatures, $k_{\mathrm{B}} T \geqslant\left\langle E_{\mathrm{ph}}\right\rangle$ and the slope of the $E_{\mathrm{g}}(T)$ curve approaches its limiting value:

$-\left[d E_{\mathrm{g}} / d T\right]_{\max }=2 S k_{B}$

The peak energy variation with temperature, $P E(T)$, of the nearband-edge luminescence was analysed further using the three models presented before. Assuming that the band gap energy can be obtained simply by adding the free-exciton binding energy to the $\mathrm{PE}(T)$ data over the whole temperature range, we exchanged $E_{g}(T)$ with the transition energy of the near-band-edge photoluminescence $\mathrm{PE}(T)$, i.e. the free-exciton transition energy, and the fit parameter $E_{\mathrm{g}}(0)$ in all models (Eqs. (1), (2), (5) and (7)) with $\mathrm{PE}(0)$. The fitting plots of the Einstein model (Eq. (2)) and the Pässler model (Eq. (7)) are shown in Fig. 2. The fitting plots of three-parameter thermodynamic model (Eq. (1)) and Pässler model (Eq. (5)) are close to results of model (2) and (7), respectively. For clarity we have omitted former plots from Fig. 2. The obtained fitting parameters are summarized in Table 1. By fitting our data of $P E(T)$ to Eq. (1) the following set of parameters was obtained: $P E(0)=2.500 \mathrm{eV}, S=1.53 \pm 0.12$, and $\left\langle E_{\mathrm{ph}}\right\rangle=38 \pm 3 \mathrm{meV}$. Our value of the electron-phonon coupling parameter $S$ is higher than the reported value for $\mathrm{CuGaSe}_{2}$ (1.29) [16]. It can be concluded that the interaction between band-edge states and the phonon system in $\mathrm{CuGaS}_{2}$ is stronger than in $\mathrm{CuGaSe}_{2}$

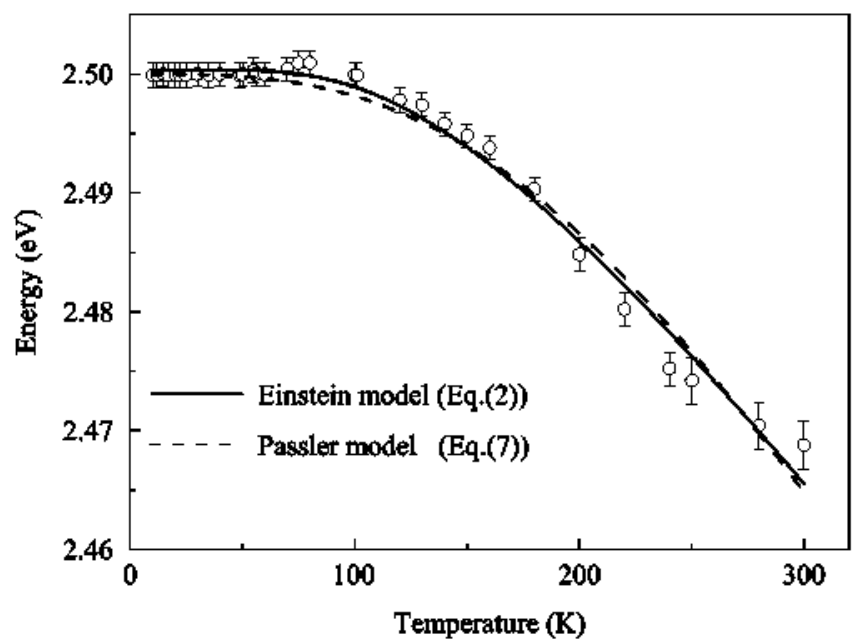

Fig. 2. Temperature dependence of the peak energy (PE) of near-band-edge luminescence, $\mathrm{PE}(T)$, in $\mathrm{CUGaS}_{2}$. The open circles are the experimentally obtained peak energies, the curves represent the calculated dependency according to the Einstein model (Eq. (2)) and the Pässler model (Eq. (7)).
Table 1

Values of the adjustable parameters $\left(\mathrm{PE}(0),\left\langle\mathbf{E}_{p h}\right\rangle, 5\right),(\mathrm{PE}(0), K, \Xi),(\mathrm{PE}(0), \alpha, \Theta, p)$ and $(\mathrm{PE}(0), \alpha, \Theta, r)$, obtained by fitting Eqs, (1), (2), (5) and (7), respectively, to the $P E(T)$ data of $\mathrm{CuGaS}_{2}$. Enors are given in parentheses.

\begin{tabular}{lll}
\hline & Parameter's & \\
\hline Eq(1) & $\mathrm{PE}(0)(\mathrm{eV})$ & 2.500 \\
& $\langle\mathrm{Eph}\rangle(\mathrm{meV})$ & $38.0(3)$ \\
& $S$ & $1.53(12)$ \\
Eq. (2) & $\mathrm{PE}(\mathrm{0})(\mathrm{eV})$ & 2.500 \\
& $K(\mathrm{eV})$ & $0.117(18)$ \\
& $\Xi(\mathrm{K})$ & $441(32)$ \\
& $\mathrm{PE}(0)(\mathrm{eV})^{\mathrm{a}}$ & 2.5 \\
Eq. (5) & $\propto(\mathrm{meV} \mathrm{K}-1)$ & $0.31(2)$ \\
& $\Theta(\mathrm{K})^{\mathrm{a}}$ & 441 \\
& $p$ & $3.1(2)$ \\
& $\mathrm{PE}(0)\left(\mathrm{eV}^{\mathrm{a}}\right.$ & 2.5 \\
& $\alpha\left(\mathrm{meV} \mathrm{K}^{-1}\right)$ & $0.32(2)$ \\
& $\Theta(\mathrm{K})^{\mathrm{a}}$ & 441 \\
& $r$ & $0.19(6)$ \\
\hline
\end{tabular}

a Fixed parameters in the fitting.

Comparing the above given $T$ asymptote of Eqs. (1) or (2) with its counterpart in Eq. (5), the following correlations between the different parameters of the models are found [16]:

$2 S k_{\mathrm{B}}=K \mid \Xi=\delta$ magnitude of the limiting slope and $\left\langle E_{\mathrm{ph}}\right\rangle \mid$ $k_{\mathrm{B}}=\Xi=\Theta$ for the effective phonon temperature. Comparing the parameter values listed in Table 1 , we conclude that these theoretical relations are satisfactorily fulfilled. The calculated value of $(\mathrm{dPE} / \mathrm{dT})_{\max }=0.26 \mathrm{meV} \mathrm{K}^{-1}$ is close to the parameter $\alpha=0.31 \pm 0.02 \mathrm{mEvK}^{-1}$ obtained by the Pässler model. Our parameters ( $(\mathrm{PE}(0)), K, \Xi$ and $\alpha)$ are in good agreement with those estimated by Shirakata and Chichibu [6] based on the Einstein model.

Assuming that mobility $\mu$ is determined by lattice scattering, its value can be expressed by [13]

$\mu=(48 / 9 \pi)(3 / 4 \pi)^{1 / 3}(\pi / 2)^{1 / 2} e h^{2} k /(\Omega)^{1 / 3} m^{* 3 / 2}\left(k_{\mathrm{B}} T\right)^{3 / 2}\left(d E_{\mathrm{g}} / d T\right)$

where $\Omega$ is the volume of the unit cell and $m^{*}$ is the effective mass. The hole mobility $\mu_{\mathrm{h}}$ and electron mobility $\mu_{\mathrm{e}}$ were calculated by assuming that the values of $m_{h} / m_{0}$ and $m_{e} / m_{0}$ are equal to 0.69 and 0.13 , respectively [20]. At room temperature we have estimated the hole and electron mobility equal to 71 and $869 \mathrm{~cm}^{2} / \mathrm{Vs}$, respectively. No previously reported data on the electron mobility in $\mathrm{CuGaS}_{2}$ has been found, whereas the available data on the hole mobility is $15 \mathrm{~cm}^{2} / \mathrm{Vs}$ [21]. The latter show a quite low room temperature value probably caused by a strong compensation of samples studied.

Eq. (5) provides a good approximation of Eq. (4) if the value of parameter $p$ is between 2.2 and 2.6. By fitting Eq. (5) to our $P E(T)$ data, the following set of parameters is obtained: $p=3.1 \pm 0.2$, and $\alpha=0.31 \pm 0.02 \mathrm{meV} \mathrm{K}^{-1}$ (Table 1 ). The value of $p$ is higher than the above-mentioned upper limit of $p=2.6$. Similar behavior is typical for wide-gap materials ( $p$ is found to be equal to 2.9 for $\mathrm{GaN}$ and to 3.1 for $\mathrm{SiC}$ ). The effective phonon energy $k_{\mathrm{B}} \Theta$ is equal to $36 \mathrm{meV}$ and the cut-off phonon energy is found to be $56 \pm 14 \mathrm{meV}$

Eq. (7) provides a good approximation of Eq. (4) if the value of parameter $r$ is higher than 0.15, which is valid for the case of our $\mathrm{CuGaS}_{2}$ data. By fitting Eq. (7) to our $E_{\mathrm{g}}(T)$ data, the following set of parameters is obtained: $r=0.19 \pm 0.06$ and $\alpha=0.32 \pm 0.02 \mathrm{meV}$ $\mathrm{K}^{-1}$ (Table 1). The effective phonon energy $k_{\mathrm{B}} \Theta$ is equal to $36 \mathrm{meV}$ and the cut-off phonon energy is found to be $43 \pm 5 \mathrm{meV}$. 
Both four-parameter representations (Eqs. (5) and (7)) allow good fitting of our experimental data on the temperature variation of the peak energy of near-band-edge luminescence; however the condition that $r$ is higher than 0.15 (Eq. (7)) is better fulfilled than $p=2.2-2.6$ (Eq. (5)) (see Table 1). At room temperature, Carlone et al [22] have reported on 22 optical phonon modes in $\mathrm{CuGaS}_{2}$ by means of the Raman spectroscopy. The frequencies of these modes vary from $75 \mathrm{~cm}^{-1}$ ( $9 \mathrm{meV}$ ) up to $401 \mathrm{~cm}^{-1}$ ( $49 \mathrm{meV}$ ).

The cut-off phonon energy, as estimated from the Pässler model, is close to the highest frequency modes observed in the Raman and $I R$ investigations, and the difference is within the accuracy of the data. It is worth mentioning that Eq. (7) permits one to estimate the cut-off phonon energy with higher accuracy than Eq. (5).

The mean frequency of the vibrational modes estimated by considering the Raman-active optical modes reported in Ref. [20] is equal to $243 \mathrm{~cm}^{-1}$ ( $30 \mathrm{meV}$ ), which is in agreement with the effective phonon energy $k_{\mathrm{B}} \Theta=36 \mathrm{meV}$ obtained from Pässler's model. This leads to the conclusion that the major contribution of phonons to the shift of the peak energy with temperature in $\mathrm{CuGaS}_{2}$ comes mainly from optical phonons.

Adding a minimum free-exciton binding energy of $29 \mathrm{meV}$ [6], the minimum band gap energy at $T=0 \mathrm{~K}$ is estimated to be $2.5293 \mathrm{eV}$ and, thus, it is in agreement with recently reported values (2.53 eV at $5 \mathrm{~K} \mathrm{[4]} \mathrm{and} 2.497 \mathrm{eV}$ at $20 \mathrm{~K}$ [5])

\section{Conclusions}

The photoluminescence of $\mathrm{CuGaS}_{2}$ single crystals has been investigated. The temperature dependence of the near-band-edge $\mathrm{PL}$, reflecting the temperature dependence of the excitonic band gap in the temperature range of $10-300 \mathrm{~K}$, was studied by means of a three-parameter thermodynamic model, the Einstein model and the Pässler model. All models used provide good fits to our experimental data on temperature variation of the band gap within the accuracy of the measurement. The values of the band gap at $T=0 \mathrm{~K}$, the effective and cut-off phonon energy, a dimensionless constant related to the electron-phonon coupling and an estimation for the room-temperature electron (hole) mobility of $\mathrm{CuGaS}_{2}$ have been derived from the applied models. The coincidence of the effective phonon energy obtained from the Pässler's model with mean energy estimated from Raman-active reported optical modes leads to the conclusion that the major contribution of phonons to the shift of $E_{g}$ with $T$ in $\mathrm{CuGaS}_{2}$ is mainly from optical phonons.

\section{Acknowledgment}

The authors would like to thank Dr. A. Meeder for his help in experiments and useful discussions.

\section{References}

[1] ]. Repins, M.A. Contreras, B. Egaas, C. DeHart. J. Scharf, C.l. Perkins, B. To, R. Noufi, Prog. Photovolt. 16 (2008) 235.

[2] J.L. Shay, B. Tell, H.M. Kasper, Appl. Phys. lett. 19 (1971) 366.

[3] H. Metzner, Tl. Hahn, J. Cieslak, U, Grossner, U. Reislohner, W. Witthulın, R. Goldhahn, J. Eberhardt, G. Gobsch, J. Krausslich, Appl. Phys. Lett. 81 (2002) 156 .

[4] C. Bellababa, J. Gonzalez, C. Rincon, Phys. Rev. B 53 (1996) 7792.

[5] M.J. Alonso, K. Wakita, J. Pascual, M. Garriga, N. Yamamoto, Phys. Rev. B 63 (2001) 075203 .

[6] S. Shirakata, S. Chichibu, J. Appl. Phys. 87 (2000) 3793.

[7] S. Shirakata, K. Saiki, 5. Isomura, J. Appl. Phys. 68 (1990) 291.

[8] J. Eberhardt, H. Metzner, Th. Hahn, U. Reislohner, J. Cieslak, U. Grossner, R. Goldhahn, F. Hudert, G. Gobsch, W. Witthuhn, J. Phys. Chem. Sol. 64 (2003) 1781 .

[9] H. Metzner, J. Eberhardt, J. Cieslak, Th. Hahn, R. Goldhahn, U. Reislohner, W. Witthulm. Thin Solid Films 451-452 (2004) 241.

[10] N.N. Syrbu, V.V. Ursaki, I.M. Tiginyanu, V.E. Tezlevan, M.A. Blaje, J. Pliys Chem. Sol. 64 (2003) 1967.

[11] H. Metzner, J. Cieslak, U. Grossner, Th. Hahn, U, Kaiser, J. Krausslich, U. Reislohner, W. Witthuhn, R. Goldhahn, J. Eberhardt, Thin Soljd Films 431-432 (2003) 219 .

[12] ].-H. Choi, P.Y. Yu, ]. Phys, Chem. Solids 51 (1996) 1695.

[13] K.P. O'Donnel, X. Chen, Appl. Phys. Lett. 58 (1991) 2924

[14] Z. Yang. K.P. Homewood, M.S. Finney. M.A. Harry. J. Reeson, J. Appl. Phys. 78 (1995) 1958.

[15] R. Pässler, Pliys. 5tatus Solidi B 200 (1997) 155

[16] A. Meeder, A. Jager-Waldau, V. Tezlevan, E. Alushanov, T. 5chidel-Niedrig. M.C.h lux-Steiner, J. Phys: Condens. Matter 15 (2003) 6219.

[17] E. Arushanov, Cl. Bucher, O. Kloc, l. Kuljkova, Kulyuk, A. Simine], Plivs. Rev, B 52 (1995) 20.

[18] S. levcenko. N.N. Syrbu, A. Nateprov, E. Arushanov, J.M. Merino, M. León, J Phys. D: Appl. Plys, 39 (2006) 1515

[19] S. Levcenko, N.N. Syrbu, E. Arushanov, V. Tezlevan, R. Fernández-Ruiz, J.M. Merino. M. león. J. Appl. Phys. 99 (2006) 073513.

[20] N. Yamomoto, H. Horinka, K. Okada, T. Miyauchi, Jpn. J. Appl. Phys. 16 (1977) 1817

[21] J.L Shay. J.H. Wernik, in: Ternary Chalcopyrite Semiconductors: Growth, Electronic Propertries and Applications.Pergamon, 1975.

[22] C. Carlone, D. Olego, A. Jayaraman, M. Cordona, Pliys. Rev, B 22 (1980) 3877. 